\title{
DIVERGENCE FORMS OF THE $\infty$-LAPLACIAN
}

\author{
Luigi D'Onofrio, Flavia Giannetti, Tadeusz Iwaniec, \\ Juan Manfredi and Teresa Radice
}

\begin{abstract}
The central theme running through our investigation is the $\infty$-Laplacian operator in the plane. Upon multiplication by a suitable function we express it in divergence form, this allows us to speak of weak $\infty$-harmonic function in $\mathscr{W}^{1,2}$. To every $\infty$-harmonic function $u$ we associate its conjugate function $v$. We focus our attention to the first order Beltrami type equation for $h=u+\imath v$.
\end{abstract}

\section{Introduction}

This paper is concerned with various linear and nonlinear PDEs whose prototype is the $p$-harmonic equation

$$
\operatorname{div}\left(|\nabla u|^{p-2} \nabla u\right)=0, \quad 1<p<\infty .
$$

The focus is on the limiting case as $p$ approaches $\infty$, referred to as the $\infty$-Laplacian

$$
\Delta_{\infty} u=2 \sum_{i, j=1}^{n} \frac{u_{x_{i}} u_{x_{j}}}{|\nabla u|^{2}} \frac{\partial^{2} u}{\partial x_{i} \partial x_{j}} .
$$

Upon multiplication by a suitable function $\lambda=\lambda(\nabla u)$ we express this operator in divergence form. There may be several such functions $\lambda=$ $\lambda(\nabla u)$. We call them divergence factors. Writing the $\infty$-Laplacian in divergence form allows to speak of weak $\infty$-harmonic functions in the Sobolev class $\mathscr{W}_{\text {loc }}^{1,2}(\Omega)$. In analogy to the Cauchy-Riemann system we associate to every $\infty$-harmonic function $u \in W_{\text {loc }}^{1,2}(\Omega)$ in the planar domain $\Omega$ its conjugate function $v$. In general the complex function $h=u+\imath$ is multivalued. This could happen because of the lack of existence of a continuous branch of the argument of $w=u_{x}+\imath u_{y}$. The

2000 Mathematics Subject Classification. Primary: 35J70; Secondary: 35B04.

Key words. $\infty$-Laplacian operator, $\infty$-harmonic functions. 
novelty of our approach is that the first order Beltrami type equation for $h$ is elliptic and, therefore, defines a mapping of finite distortion.

\section{Divergence factors and integrating fields}

When dealing with nonlinear partial differential equations, it is often convenient to write them in divergence form. Consider, for example, the question of the domain of definition of a given nonlinear differential operator. Expressing this operator in a divergence form, makes one derivative dispensable in the definition of its domain. Naturally, there may exist many divergence forms of an operator, leading to different domains of definition. A typical example is furnished by the Hessian determinant in two variables:

$$
\begin{aligned}
& \operatorname{det} \mathcal{H} u=\operatorname{det}\left[\begin{array}{ll}
u_{x x} & u_{x y} \\
u_{x y} & u_{y y}
\end{array}\right] \\
& =u_{x x} u_{y y}-u_{x y} u_{x y} \\
& \text { for } u \in \mathscr{W}_{\text {loc }}^{2,2}(\Omega) \\
& =\left(u_{x} u_{y y}\right)_{x}-\left(u_{x} u_{x y}\right)_{y} \quad \text { for } \quad u \in \mathscr{W}_{\operatorname{loc}}^{2,4 / 3}(\Omega) \\
& =\frac{1}{2}\left(u u_{x x}\right)_{y y}+\frac{1}{2}\left(u u_{y y}\right)_{x x}-\left(u u_{x y}\right)_{x y} \quad \text { for } \quad u \in \mathscr{W}_{\text {loc }}^{2,1}(\Omega) \\
& =\left(u_{x} u_{y}\right)_{x y}-\frac{1}{2}\left(u_{x} u_{x}\right)_{y y}-\frac{1}{2}\left(u_{y} u_{y}\right)_{x x} \quad \text { for } \quad u \in \mathscr{W}_{\operatorname{loc}}^{1,2}(\Omega) .
\end{aligned}
$$

In another example, the reader may try to verify the following identity for the Gaussian curvature of a surface $z=u(x, y)$ in $\mathbb{R}^{3}$,

$$
\mathcal{K} u=\frac{u_{x x} u_{y y}-u_{x y} u_{x y}}{\left(1+u_{x}^{2}+u_{y}^{2}\right)^{2}}=\frac{\operatorname{det} \mathcal{H} u}{\left(1+|\nabla u|^{2}\right)^{2}} .
$$

First notice that $\mathcal{K} u$ is none other than the Jacobian determinant of the mapping

$$
\begin{gathered}
(A, B)=\left(\frac{u_{x}}{\sqrt{1+u_{x}^{2}+u_{y}^{2}}}, \frac{u_{y}}{\sqrt{1+u_{x}^{2}+u_{y}^{2}}}\right), \\
\mathcal{K}=A_{x} B_{y}-A_{y} B_{x} .
\end{gathered}
$$

We can express $\mathcal{K} u$ in divergence form using two different formulas

$$
\mathcal{K}=\left(A B_{y}\right)_{x}-\left(A B_{x}\right)_{y}=\left(A_{x} B\right)_{y}-\left(A_{y} B\right)_{x} .
$$

This leads us to two different divergence forms of the curvature

$$
\mathcal{K}=\operatorname{div} \mathbf{F} .
$$


The so-called integrating field $\mathbf{F}=\left(F^{1}, F^{2}\right)$ can be expressed as

$$
\left\{\begin{array}{l}
F^{1}=\frac{u_{x}}{\left(1+u_{x}^{2}+u_{y}^{2}\right)^{2}}\left[\left(1+u_{x}^{2}\right) u_{y y}-u_{x} u_{y} u_{x y}\right] \\
F^{2}=-\frac{u_{x}}{\left(1+u_{x}^{2}+u_{y}^{2}\right)^{2}}\left[\left(1+u_{x}^{2}\right) u_{x y}-u_{x} u_{y} u_{x x}\right]
\end{array}\right.
$$

or

$$
\left\{\begin{array}{l}
F^{1}=-\frac{u_{y}}{\left(1+u_{x}^{2}+u_{y}^{2}\right)^{2}}\left[\left(1+u_{y}^{2}\right) u_{x y}-u_{x} u_{y} u_{y y}\right] \\
F^{2}=\frac{u_{y}}{\left(1+u_{x}^{2}+u_{y}^{2}\right)^{2}}\left[\left(1+u_{y}^{2}\right) u_{x x}-u_{x} u_{y} u_{x y}\right]
\end{array} .\right.
$$

Adding up these two solutions we gain a symmetry with respect to $x$ and $y$. Namely,

$$
\begin{aligned}
2 \mathcal{K} & =\left(A B_{y}-A_{y} B\right)_{x}+\left(A_{x} B-A B_{x}\right)_{y} \\
2 \mathbf{F} & =\left(\frac{u_{x} u_{y y}-u_{y} u_{x y}}{1+u_{x}^{2}+u_{y}^{2}}, \frac{u_{y} u_{x x}-u_{x} u_{x y}}{1+u_{x}^{2}+u_{y}^{2}}\right) .
\end{aligned}
$$

One interesting outcome of this calculation is that the Gaussian curvature can be defined for surfaces parameterized by functions in $\mathscr{W}_{\text {loc }}^{2,1}(\Omega)$. Such parametrizations have integrating factors $\mathbf{F} \in \mathscr{L}_{\text {loc }}^{1}\left(\Omega, \mathbb{R}^{n}\right)$.

In this paper we are concerned with the differential operators which are linear with respect to the second order derivatives,

$$
\mathcal{L} u=\sum_{i, j=1}^{n} G_{i j}(\nabla u) \frac{\partial^{2} u}{\partial x_{i} \partial x_{j}} .
$$

Here the symmetric matrix function

$$
\mathbf{G}=\mathbf{G}(V)=\left[G_{i j}(V)\right], \quad i, j=1, \ldots, n
$$

defined for $V \in \mathbb{R}^{n}$ may have a singularity at $V=0$. This corresponds to the critical points of $u$. Let us try to express $\mathcal{L} u$ in divergence form

$$
\mathcal{L} u=\operatorname{div} \mathbf{F}(\nabla u), \quad \mathbf{F}=\left(F^{1}, \ldots, F^{n}\right) .
$$

Elementary computations show that the integrating field $\mathbf{F}: \mathbb{R}^{n} \backslash\{0\} \rightarrow$ $\mathbb{R}^{n}$ must satisfy the following overdetermined system of PDEs

$$
\mathrm{D}^{*} \mathbf{F}(V)+\mathrm{D} \mathbf{F}(V)=2 \mathbf{G}(V) .
$$

The solvability conditions for such systems of PDEs are well known in the calculus of differential forms. One particular case arises naturally in linear elasticity,

$$
\mathrm{D}^{*} \mathbf{F}+\mathrm{D} \mathbf{F}-\frac{2}{n}(\operatorname{Tr} \mathrm{D} \mathbf{F}) I=\mathbf{C}, \quad \operatorname{Tr} \mathbf{C} \equiv 0
$$


where $\mathbf{C}=2 \mathbf{G}-\frac{2}{n}(\operatorname{Tr} \mathbf{G}) I$. This system also results from infinitesimal deformations of the nonlinear Beltrami equation

$$
(I+\varepsilon \mathrm{D} \mathbf{F})^{*}(I+\varepsilon \mathrm{DF})=[\operatorname{det}(I+\varepsilon \mathrm{DF})]^{\frac{2}{n}}(I+\varepsilon \mathbf{C}) .
$$

The solvability conditions for the system (2.2) have been completely identified by L. V. Ahlfors $[\mathbf{A}],[\mathbf{A 1}]$.

Consider the simplest second order differential operator

$$
\mathcal{L} u=\Delta u, \quad \mathbf{G}=I .
$$

Obviously it has the divergence form

$$
\Delta u=\operatorname{div}[\mathbf{F}(\nabla u)]
$$

where $\mathbf{F}(V)=V$. There are, however, other divergence forms of the Laplacian once we multiply it by a suitable factor. For example,

$u_{x_{1}} \Delta u=\frac{1}{2}\left[\left(u_{x_{1}}^{2}-u_{x_{2}}^{2}-\cdots-u_{x_{n}}^{2}\right)_{x_{1}}+\left(2 u_{x_{1}} u_{x_{2}}\right)_{x_{2}}+\cdots+\left(2 u_{x_{1}} u_{x_{n}}\right)_{x_{n}}\right]$.

This corresponds to the following integrating field

$$
\mathbf{F}(V)=\left(v_{1}^{2}-v_{2}^{2}-\cdots-v_{n}^{2}, 2 v_{1} v_{2}, \ldots, 2 v_{1} v_{n}\right)
$$

which satisfies the equation

$$
\mathrm{D}^{*} \mathbf{F}(V)+\mathrm{D} \mathbf{F}(V)=2 v_{1} I, \quad V=\left(v_{1}, v_{2}, \ldots, v_{n}\right) .
$$

We say that $u_{x_{1}}$ is a divergence factor for the Laplacian. Similarly, $u_{x_{2}}, \ldots, u_{x_{n}}$ are also divergence factors. As before, we combine those equations into one symmetric form.

Example 2.1. The vector valued function $\lambda=\nabla u$ is a divergence factor of the Laplacian. Precisely, we have

$$
(\nabla u) \Delta u=\operatorname{Div}\left[\nabla u \otimes \nabla u-\frac{1}{2},|\nabla u|^{2} I\right] .
$$

The operator Div applies to the row vectors of the matrix in the right hand side, resulting in a vector field. What we gain here is not only symmetry but also the fact that the divergence factor vanishes only at the critical points of $u$; that is, when $\nabla u=0$. Having disposed of these preliminary examples we are now ready to formulate a more general concept of divergence factor and associated integrating field.

In what follows $\mathbb{E}$ is a finite dimensional linear space.

Definition 2.2. A divergence factor of the differential operator $\mathcal{L}$ is a function $\lambda: \mathbb{R}^{n} \backslash\{0\} \rightarrow \mathbb{E}$ for which there exists $\mathbf{F}: \mathbb{R}^{n} \backslash\{0\} \rightarrow \mathbb{R}^{n} \otimes \mathbb{E}$, called an integrating field, such that

$$
\lambda(\nabla u) \mathcal{L} u=\operatorname{Div}[\mathbf{F}(\nabla u)], \quad \text { at noncritical points of } u .
$$




\section{The $p$-Laplacian}

The $p$-harmonic equation

$$
\operatorname{div}|\nabla u|^{p-2} \nabla u=0
$$

is the Euler-Lagrange equation of the variational integral

$$
\mathcal{E}_{p}[u]=\frac{1}{p} \int_{\Omega}|\nabla u(x)|^{p} \mathrm{~d} x, \quad 1<p<\infty .
$$

That is why the Sobolev space $\mathscr{W}^{1, p}(\Omega)$ is viewed as the natural domain of definition of this equation. However, this equation can also be expressed as a fully non-linear equation, in nondivergence form, by carrying out the differentiation

$$
|\nabla u|^{2} \Delta u+(p-2) \sum_{i=1}^{n} \sum_{j=1}^{n} u_{x_{i}} u_{x_{j}} u_{x_{i} x_{j}}=0
$$

Although it would appear that (3.2) requires $u$ to have second order derivatives, this equation can also be interpreted in the viscosity sense. In fact the notions of weak solution of (3.1) and viscosity solution of (3.2) coincide, see [JLM2].

The border line exponents $p=1$ and $p=\infty$ can also be considered. We set

$$
\Delta_{\infty} u \stackrel{\text { def }}{=} 2 \sum_{i, j=1}^{n} \frac{u_{x_{i}} u_{x_{j}}}{|\nabla u|^{2}} \frac{\partial^{2} u}{\partial x_{i} \partial x_{j}}=\frac{2}{|\nabla u|^{2}} \operatorname{Tr}(\nabla u \otimes \nabla u) \mathcal{H} u
$$

and

$\Delta_{1} u \stackrel{\text { def }}{=} 2 \sum_{i, j=1}^{n}\left(\delta_{i}^{j}-\frac{u_{x_{i}} u_{x_{j}}}{|\nabla u|^{2}}\right) \frac{\partial^{2} u}{\partial x_{i} \partial x_{j}}=\frac{2}{|\nabla u|^{2}} \operatorname{Tr}\left(|\nabla u|^{2} I-\nabla u \otimes \nabla u\right) \mathcal{H} u$.

The $p$-Laplacian is then a linear combination of $\Delta_{1}$ and $\Delta_{\infty}$,

$$
\Delta_{p}=\frac{1}{p} \Delta_{1}+\frac{p-1}{p} \Delta_{\infty}
$$

More explicitly we have

$$
\begin{aligned}
\Delta_{p} u & =\frac{2}{p} \sum_{i, j=1}^{n}\left(\delta_{i}^{j}+(p-2) \frac{u_{x_{i}} u_{x_{j}}}{|\nabla u|^{2}}\right) \frac{\partial^{2} u}{\partial x_{i} \partial x_{j}} \\
& =\frac{2}{p|\nabla u|^{2}} \operatorname{Tr}\left(|\nabla u|^{2} I+(p-2) \nabla u \otimes \nabla u\right) \mathcal{H} u .
\end{aligned}
$$


Thus, the scalar function $\lambda=|\nabla u|^{p-2}$ is a divergence factor of $\Delta_{p} u$. Precisely we have

$$
|\nabla u|^{p-2} \Delta_{p} u=\frac{2}{p} \operatorname{div}|\nabla u|^{p-2} \nabla u .
$$

The corresponding integrating field equals $\mathbf{F}(V)=|V|^{p-2} V$. Indeed,

$$
\mathrm{DF}=|V|^{p-2}\left(I+(p-2) \frac{V \otimes V}{|V|^{2}}\right) .
$$

Hence (2.1) holds with

$$
\mathbf{G}(V)=|V|^{p-2}\left(I+(p-2) \frac{V \otimes V}{|V|^{2}}\right) .
$$

It is worth noting that no other power function of the form $|V|^{k} V$ can be an integrating field for the $p$-Laplacian. However, there exist more sophisticated integrating fields.

\section{The $p$-harmonic equation in the plane}

The class of divergence factors is particularly rich in two dimensions due to the complex structure in $\mathbb{R}^{2} \cong \mathbb{C}=\{z=x+\imath y, x, y \in \mathbb{R}\}$. Let $\Omega$ be an open subset of the complex plane. A function $u \in \mathscr{C}^{2}(\Omega)$ is:

- $\infty$-harmonic if

$$
\frac{1}{2}|\nabla u|^{2} \Delta_{\infty} u=u_{x x} u_{x}^{2}+2 u_{x y} u_{x} u_{y}+u_{y y} u_{y}^{2}=0,
$$

- 1-harmonic if

$$
\frac{1}{2}|\nabla u|^{2} \Delta_{1} u=u_{x x} u_{y}^{2}-2 u_{x y} u_{x} u_{y}+u_{y y} u_{x}^{2}=0,
$$

and

- $p$-harmonic if

(4.3) $\frac{1}{2}|\nabla u|^{2} \Delta_{p} u=\frac{1}{p}|\nabla u|^{2} \Delta u+\left(1-\frac{2}{p}\right)\left(u_{x x} u_{x}^{2}+2 u_{x y} u_{x} u_{y}+u_{y y} u_{y}^{2}\right)=0$.

We shall make use of the Cauchy-Riemann derivatives

$$
\frac{\partial}{\partial z}=\frac{1}{2}\left(\frac{\partial}{\partial x}-\imath \frac{\partial}{\partial y}\right) \text { and } \frac{\partial}{\partial \bar{z}}=\frac{1}{2}\left(\frac{\partial}{\partial x}+\imath \frac{\partial}{\partial y}\right)
$$

and the complex gradient of $u$, which is defined by

$$
f(z)=u_{z}=\frac{1}{2}\left(u_{x}-\imath u_{y}\right) .
$$


The $p$-Laplacian of $u$ can be expressed in terms of $f$ as

$$
\frac{1}{4} \Delta_{p} u=\frac{\partial f}{\partial \bar{z}}+\left(\frac{1}{2}-\frac{1}{p}\right)\left[\bar{f} \frac{\partial f}{\partial z}+\frac{f}{\bar{f}} \frac{\overline{\partial f}}{\partial z}\right] .
$$

This is an elliptic operator for all $1<p<\infty$. However, the borderline cases lead to formally parabolic operators

$$
\frac{1}{4} \Delta_{1} u=\frac{\partial f}{\partial \bar{z}}-\frac{1}{2}\left[\frac{\bar{f}}{f} \frac{\partial f}{\partial z}+\frac{f}{\bar{f}} \frac{\overline{\partial f}}{\partial z}\right]
$$

and

$$
\frac{1}{4} \Delta_{\infty} u=\frac{\partial f}{\partial \bar{z}}+\frac{1}{2}\left[\frac{\bar{f}}{f} \frac{\partial f}{\partial z}+\frac{f}{\bar{f}} \frac{\overline{\partial f}}{\partial z}\right]
$$

We can view the complex gradient of the $p$-harmonic function as a solution of the Beltrami equation

$$
f_{\bar{z}}=\mu(z) f_{z}, \quad \mu(z)=\left(\frac{1}{p}-\frac{1}{2}\right)\left[\frac{\bar{f}}{f}+\frac{f}{\bar{f}} \frac{\overline{f_{z}}}{f_{z}}\right]
$$

which is always elliptic if $1<p<\infty$. For $p=1$ and $p=+\infty$ we observe that the distortion function $K(z)=\frac{1+|\mu(z)|}{1-|\mu(z)|}$ is still finite at the points where

$$
\bar{f} \frac{\partial f}{\partial z} \notin \mathbb{R}
$$

At these points the equation (4.5) remains elliptic.

Of particular interest to us will be the complex gradients of $\infty$-harmonic functions. These are solutions of the quasilinear first order system

$$
\frac{\partial f}{\partial \bar{z}}=-\Re e\left(\frac{\bar{f}}{f} \cdot \frac{\partial f}{\partial z}\right)=-\frac{1}{2}\left(\frac{\bar{f}}{f} \cdot \frac{\partial f}{\partial z}+\frac{f}{\bar{f}} \cdot \frac{\overline{\partial f}}{\partial z}\right) .
$$

The Jacobian determinant of $f$ is computed as:

$$
\mathcal{J}(z, f)=\left|f_{z}\right|^{2}-\left|f_{\bar{z}}\right|^{2}=\left|\Im m \frac{\bar{f}}{f} \cdot \frac{\partial f}{\partial z}\right|^{2} .
$$

Thus $\mathcal{J}(z, f)$ is positive at the points where (4.6) is elliptic. 


\section{The hodograph transformation}

Assuming that the complex gradient is a homeomorphism, we can solve the equation $\xi=f(z)$ for $z \stackrel{\text { def }}{=} \Phi(\xi)$. In this connection we remind the paper [IMa]. The chain rule gives

$$
f_{z}=\frac{-\overline{\Phi_{\xi}}}{\mathcal{J}(\xi, \Phi)} \quad \text { and } \quad f_{\bar{z}}=\frac{\Phi_{\bar{\xi}}}{\mathcal{J}(\xi, \Phi)} .
$$

Equation (4.3) becomes linear in $\Phi$

(5.2) $\frac{\partial \Phi}{\partial \bar{\xi}}=\left(1-\frac{2}{p}\right) \Re e\left(\frac{\xi}{\bar{\xi}} \cdot \frac{\partial \Phi}{\partial \xi}\right)=\left(\frac{1}{2}-\frac{1}{p}\right)\left(\frac{\xi}{\bar{\xi}} \cdot \frac{\partial \Phi}{\partial \xi}+\frac{\bar{\xi}}{\xi} \cdot \frac{\overline{\partial \Phi}}{\partial \xi}\right)$.

In particular, for $p=\infty$ and $p=1$, we have

$$
\frac{\partial \Phi}{\partial \bar{\xi}}= \pm \Re e\left(\frac{\xi}{\bar{\xi}} \cdot \frac{\partial \Phi}{\partial \xi}\right)= \pm \frac{1}{2}\left(\frac{\xi}{\bar{\xi}} \cdot \frac{\partial \Phi}{\partial \xi}+\frac{\bar{\xi}}{\xi} \cdot \frac{\overline{\partial \Phi}}{\partial \xi}\right),
$$

where the + sign corresponds to $p=\infty$ and the - sign corresponds to $p=1$. A family of basic solutions for the + sign is described in the following

Proposition 5.1. For $k=0,1,2, \ldots$ and $\gamma_{k} \in \mathbb{C}$, the functions

$$
\Phi_{k}(\xi)=|\xi|^{k^{2}-1}\left[(k-1) \bar{\gamma}_{k}\left(\frac{\bar{\xi}}{|\xi|}\right)^{k+1}+(k+1) \gamma_{k}\left(\frac{\xi}{|\xi|}\right)^{k-1}\right]
$$

solve the system (5.3) with the + sign.

It is worth noting that these formulas still define (multivalued) solutions when $k$ is a real parameter. Here are some particular cases

$$
\begin{aligned}
\Phi_{0}(\xi) & =\frac{\gamma}{\xi}, & & \gamma \in \imath \mathbb{R}, \\
\Phi_{1}(\xi) & =\gamma, & & \gamma \in \mathbb{C},
\end{aligned}
$$

and

$$
\Phi_{2}(\xi)=\left\{\begin{array}{l}
3|\xi|^{2} \xi+\bar{\xi}^{3} \\
\text { or } \\
\imath\left(3|\xi|^{2} \xi-\bar{\xi}^{3}\right)
\end{array} .\right.
$$

More general solutions are infinite sums of the basic ones

$$
\Phi(\xi)=\sum_{k=1}^{\infty} a_{k} \Phi_{k}(\xi), \quad a_{k} \in \mathbb{R} .
$$


Let us examine the solution $\Phi_{2}(\xi)=3|\xi|^{2} \xi+\bar{\xi}^{3}$. Using real and imaginary part of $\xi=a+\imath b$, we see that $\Phi_{2}(a+\imath b)=4 a^{3}+4 \imath b^{3}$. It is a polynomial of degree 3 , and it is a homeomorphism of the entire complex plane. In order to recover the original $\infty$-harmonic function we compute the inverse of $\Phi_{2}$

$$
f(z)=\frac{1}{2}(\sqrt[3]{2 x}+\imath \sqrt[3]{2 y}), \quad z=x+\imath y
$$

that is,

$$
u_{x}=\sqrt[3]{2 x} \quad \text { and } \quad u_{y}=-\sqrt[3]{2 y} .
$$

We integrate this system to find $u$,

$$
u=\frac{3}{4}(x \sqrt[3]{2 x}-y \sqrt[3]{2 y}) .
$$

This is an $\infty$-harmonic function of class $\mathscr{C}^{1, \alpha}$ with $\alpha=\frac{1}{3}$ first discovered by Aronsson $[\mathbf{A r}]$. It is widely believed that

Conjecture 5.1. All $\infty$-harmonic functions lie in $\mathscr{C}_{\mathrm{loc}}^{1, \frac{1}{3}}(\Omega)$.

Very recently O. Savin $[\mathbf{S}]$ has proved that $\infty$-harmonic functions in the plane are indeed $C_{\mathrm{loc}}^{1}(\Omega)$.

\section{Divergence factors for $\infty$-Laplacian}

To define $\infty$-Laplacian in the weak sense, in contrast to the viscosity sense, for functions having only first order derivatives we need to express $\Delta_{\infty}$ in a divergence form. Let us find all divergence factors $\lambda=\lambda\left(u_{x}, u_{y}\right)$ of the equation (4.1). That is, we are looking for solutions to

(6.1) $\lambda u_{x}^{2} u_{x x}+2 \lambda u_{x} u_{y} u_{x y}+\lambda u_{y}^{2} u_{y y}=\frac{\partial}{\partial x} \mathscr{A}\left(u_{x}, u_{y}\right)+\frac{\partial}{\partial y} \mathscr{B}\left(u_{x}, u_{y}\right)$.

This identity holds if and only if

$$
\frac{\partial \mathscr{A}}{\partial u_{x}}=\lambda u_{x}^{2}, \quad \frac{\partial \mathscr{B}}{\partial u_{y}}=\lambda u_{y}^{2}
$$

and

$$
\frac{\partial \mathscr{A}}{\partial u_{y}}+\frac{\partial \mathscr{B}}{\partial u_{x}}=2 \lambda u_{x} u_{y} .
$$

It will be advantageous to work with the complex function

$$
\mathscr{F}=\mathscr{F}(w) \stackrel{\text { def }}{=} \mathscr{A}+\imath \mathscr{B},
$$


of the complex variable $w=u_{x}+\imath u_{y}$. In this notation the system takes the form

$$
\frac{\partial \mathscr{F}}{\partial \bar{w}}=\frac{1}{2} \frac{w}{\bar{w}}\left(\frac{\partial \mathscr{F}}{\partial w}+\frac{\overline{\partial \mathscr{F}}}{\partial w}\right)=\frac{w}{\bar{w}} \Re e \frac{\partial \mathscr{F}}{\partial w}
$$

and

$$
\lambda=\frac{2 \mathscr{F}_{\bar{w}}}{w^{2}}=\frac{2 \Re e \mathscr{F}_{w}}{|w|^{2}} .
$$

Observe that $\mathscr{F}$ is orientation preserving in the sense that $\left|\mathscr{F}_{w}\right|^{2}-$ $\left|\mathscr{F}_{\bar{w}}\right|^{2}=\left|\mathscr{F}_{w}\right|^{2}-\left|\Re e \mathscr{F}_{w}\right|^{2}=\left|\Im m \mathscr{F}_{w}\right|^{2} \geqslant 0$.

Proposition 6.1. All complex integrating fields of the $\infty$-Laplacian are determined from the equation (6.4). The associated divergence factor is the real part of $\mathscr{F}_{w}$.

We close this section by observing that equations (6.4) and (5.3) are dual to each other via the complex inversion of variables

$$
\xi \cdot w=1 .
$$

Precisely this means that the solutions $\Phi=\Phi(\xi)$ at (5.3) and the solutions $\mathscr{F}=\mathscr{F}(w)$ of $(6.4)$ are coupled by the relations

$$
\mathscr{F}(w)=\Phi(\xi), \quad \xi \cdot w=1 .
$$

\section{Basic examples}

Using the solutions listed at (5.4) we obtain the dual system of solutions of $(6.4)$

$$
\mathscr{F}_{k}(w)=\frac{(k-1) \gamma_{k}\left(\frac{w}{|w|}\right)^{k+1}+(k+1) \overline{\gamma_{k}}\left(\frac{\bar{w}}{|w|}\right)^{k-1}}{|w|^{k^{2}-1}}
$$

for $k=0,1,2, \ldots$. The corresponding divergence factors of (7.1) are

$$
\begin{aligned}
\lambda_{k}(w) & =\frac{2 \Re e \mathscr{F}_{k}(w)}{|w|^{2}} \\
& =-\frac{(k-1) k(k+1)}{|w|^{k^{2}+2}}\left[\gamma_{k}\left(\frac{w}{|w|}\right)^{k}+\overline{\gamma_{k}}\left(\frac{\bar{w}}{|w|}\right)^{k}\right] \\
& =\frac{a_{k} \cos k \theta+b_{k} \sin k \theta}{r^{k^{2}+2}}, \quad a_{k}, b_{k} \in \mathbb{R}
\end{aligned}
$$


where $w=r e^{\imath \theta}$. In particular, the real valued functions

$$
\lambda(w)=\frac{\cos k \theta}{r^{k^{2}+2}} \quad \text { and } \quad \lambda(w)=\frac{\sin k \theta}{r^{k^{2}+2}}
$$

are divergence factors of the operator (4.1). We may, therefore, introduce the complex divergence factors

$$
\Lambda_{k}(w)=\frac{\cos k \theta+\imath \sin k \theta}{r^{k^{2}+2}}=\frac{e^{\imath k \theta}}{r^{k^{2}+2}}=\frac{w^{k}}{|w|^{k^{2}+k+2}} .
$$

The case $k=0$ gives $\mathscr{F}_{0}(w)=\gamma w$, where $\gamma \in \imath \mathbb{R}$. Hence the divergence factor is trivial, $\lambda=0$. For $k=1$ we obtain $\mathscr{F}_{1} \equiv \gamma \in \mathbb{C}$, so again $\lambda=0$. The first nontrivial case occurs when $k=2$

$$
\mathscr{F}_{2}(w)=\frac{\gamma w^{3}+3 \overline{\gamma w}|w|^{2}}{|w|^{6}}=\Phi_{2}\left(\frac{1}{w}\right) .
$$

Next we look for one solution of particular interest to us by studying the limiting case of (7.1) in which $k$ is considered as real parameter approaching zero. Let $\gamma_{k}=1$, so that $\mathscr{F}_{0}(w)=0$. Then we have

$$
\frac{\mathscr{F}_{k}(w)}{2 k}=\frac{(k-1) e^{\imath(k+1) \theta}+(k+1) e^{-\imath(k-1) \theta}}{2 k r^{k^{2}-1}} .
$$

Therefore, we can compute the limit

$$
\lim _{k \rightarrow 0} \frac{\mathscr{F}_{k}(w)}{2 k}=w(1-\imath \operatorname{Arg} w) .
$$

Thus

$$
\mathscr{F}(w)=w(1-\imath \operatorname{Arg} w)
$$

might be a solution to (6.4), in any simply connected subset of $\mathbb{C}$ $\{0\}$. Note that choosing a different branch of $\operatorname{Arg} w$ will not affect the equation (6.4) since $\imath w$ is also a solution. Direct computations reveal that indeed (7.7) is an integrating field:

$$
\frac{\partial \operatorname{Arg} w}{\partial w}=\frac{-\imath}{2 w}
$$

and

$$
\frac{\partial \operatorname{Arg} w}{\partial \bar{w}}=\frac{\imath}{2 \bar{w}} .
$$

Hence, we obtain

$$
\frac{\partial \mathscr{F}}{\partial w}=\frac{1}{2}-\imath \operatorname{Arg} w .
$$


We find that

$$
2 \frac{\partial \mathscr{F}}{\partial \bar{w}}=\frac{w}{\bar{w}} \quad \text { and } \quad \frac{\partial \mathscr{F}}{\partial w}+\frac{\overline{\partial \mathscr{F}}}{\partial w}=1
$$

as desired. The corresponding divergence factor is

$$
\lambda=\frac{2 \mathscr{F} \bar{w}}{w^{2}}=\frac{1}{|w|^{2}}
$$

Proposition 7.1. The $\infty$-Laplacian has a divergence form in which the integrating field $\mathscr{F}(w)=w(1-\imath \operatorname{Arg} w)$ is multivalued.

In other words the 2-form

$$
\left(\frac{u_{x}^{2}}{u_{x}^{2}+u_{y}^{2}} u_{x x}+2 \frac{u_{x} u_{y}}{u_{x}^{2}+u_{y}^{2}} u_{x y}+\frac{u_{y}^{2}}{u_{x}^{2}+u_{y}^{2}} u_{y y}\right) \mathrm{d} x \wedge \mathrm{d} y
$$

is locally exact and equals

$$
\mathrm{d}\left[\mathscr{A}\left(u_{x}, u_{y}\right) \mathrm{d} y-\mathscr{B}\left(u_{x}, u_{y}\right) \mathrm{d} x\right],
$$

where

$$
\begin{aligned}
& \mathscr{A}\left(u_{x}, u_{y}\right)=u_{x}+u_{y} \tan ^{-1} \frac{u_{y}}{u_{x}} \\
& \mathscr{B}\left(u_{x}, u_{y}\right)=u_{y}-u_{x} \tan ^{-1} \frac{u_{y}}{u_{x}} .
\end{aligned}
$$

The Jacobian determinant of $\mathscr{F}=\mathscr{A}+\imath \mathscr{B}$ is positive as long as $\operatorname{Arg} w \neq 0$. Indeed, we note that $\left|\mathscr{F}_{w}\right|^{2}-\left|\mathscr{F}_{\bar{w}}\right|^{2}=\left|\frac{1}{2}-\imath \operatorname{Arg} w\right|^{2}-\left|\frac{1}{2}\right|^{2}=(\operatorname{Arg} w)^{2}>0$. Another example of the divergence form of the equation (4.1) is obtained by taking into consideration the solution

$$
\mathscr{F}_{2}(w)=\Phi_{2}\left(\frac{1}{w}\right)=\frac{3}{w^{2} \bar{w}}+\frac{1}{\bar{w}^{3}} .
$$

Hence, we can write

$$
\begin{aligned}
\mathscr{A}+\imath \mathscr{B} & =\frac{1}{\left(u_{x}-\imath u_{y}\right)^{3}}+\frac{3}{\left(u_{x}+\imath u_{y}\right)^{2}\left(u_{x}-\imath u_{y}\right)} \\
& =\frac{4 u_{x}^{3}}{\left(u_{x}^{2}+u_{y}^{2}\right)^{3}}-\frac{4 \imath u_{y}^{3}}{\left(u_{x}^{2}+u_{y}^{2}\right)^{3}} .
\end{aligned}
$$

Thus, we have

$$
\begin{aligned}
& \mathscr{A}\left(u_{x}, u_{y}\right)=\frac{4 u_{x}^{3}}{\left(u_{x}^{2}+u_{y}^{2}\right)^{3}}, \\
& \mathscr{B}\left(u_{x}, u_{y}\right)=\frac{-4 u_{y}^{3}}{\left(u_{x}^{2}+u_{y}^{2}\right)^{3}},
\end{aligned}
$$


and

$$
\lambda=\frac{12\left(u_{y}^{2}-u_{x}^{2}\right)}{\left(u_{x}^{2}+u_{y}^{2}\right)^{4}} .
$$

We conclude this section with one interesting byproduct of our computations. According to (6.7) the function

$$
\Phi(\xi)=\frac{1+\imath \operatorname{Arg} \xi}{\xi}
$$

solves equation (5.3). This is none other then the inverse of the complex gradient $f=f(z)$ of an $\infty$-harmonic function. To compute $f(z)$ explicitly, we must solve the relation

$$
\frac{1+\imath \operatorname{Arg} \xi}{\xi}=z
$$

for $\xi$. Let $\theta=\operatorname{Arg} \xi$, so that $1+\imath \theta=\xi z$ and $\operatorname{Arg}(1+\imath \theta)=\theta+\operatorname{Arg} z$. Hence

$$
\tan ^{-1} \theta-\theta=\operatorname{Arg} z
$$

As the left hand side decreases in $\theta$ we may express $\theta$ in terms of $\operatorname{Arg} z$, say $\theta=\Theta(\operatorname{Arg} z)$. We can then conclude the existence of a multivalued $\infty$-harmonic function whose complex gradient equals

$$
f(z)=\frac{1+\imath \Theta(\operatorname{Arg} z)}{z} .
$$

\section{The conjugate functions}

To every integrating field there corresponds a conjugate function. Having written the $\infty$-Laplace equation in the divergence form

$$
\left[\mathscr{A}\left(u_{x}, u_{y}\right)\right]_{x}+\left[\mathscr{B}\left(u_{x}, u_{y}\right)\right]_{y}=0
$$

the conjugate function $v$ is defined by the rule

$$
\left\{\begin{array}{l}
\mathscr{A}\left(u_{x}, u_{y}\right)=v_{y} \\
\mathscr{B}\left(u_{x}, u_{y}\right)=-v_{x}
\end{array} .\right.
$$

Set

$$
\Delta=\left(\frac{\partial \mathscr{A}}{\partial u_{y}}+\frac{\partial \mathscr{B}}{\partial u_{x}}\right)^{2}-4 \frac{\partial \mathscr{A}}{\partial u_{x}} \frac{\partial \mathscr{B}}{\partial u_{y}} .
$$


According to the general classification of the first order nonlinear PDEs (see for example $[\mathbf{S a}]$ ), this system is:

- elliptic at the points where $\Delta<0$,

- hyperbolic at the points where $\Delta>0$,

- parabolic at the points where $\Delta=0$.

For the two examples discussed above we obtain

$$
\left\{\begin{array}{l}
\frac{4 u_{x}^{3}}{\left(u_{x}^{2}+u_{y}^{2}\right)^{3}}=v_{y} \\
\frac{4 u_{y}^{3}}{\left(u_{x}^{2}+u_{y}^{2}\right)^{3}}=v_{x}
\end{array}\right.
$$

and

$$
\left\{\begin{array}{l}
u_{x}+u_{y} \tan ^{-1} \frac{u_{y}}{u_{x}}=v_{y} \\
u_{y}-u_{x} \tan ^{-1} \frac{u_{y}}{u_{x}}=-v_{x}
\end{array} .\right.
$$

In the first example, the system is well defined outside the zeros of $\nabla u$. Both systems (8.3) and (8.4) are parabolic at every point. However, a given pair $(u, v)$ can also be considered as the solution to an elliptic system. Let us analyze this point of view in a general setting

$$
\left\{\begin{array}{l}
\mathscr{A}\left(u_{x}, u_{y}\right)=v_{y} \\
\mathscr{B}\left(u_{x}, u_{y}\right)=-v_{x}
\end{array},\right.
$$

where we recall that $\mathscr{A}+\imath \mathscr{B}=\mathscr{F}$ and $\mathscr{F} \bar{w}=\frac{1}{2} \frac{w}{\bar{w}}\left(\mathscr{F}_{w}+\overline{\mathscr{F}_{w}}\right)$. In analogy to the Cauchy-Riemann equations we introduce the complex function

$$
h(z)=u(z)+\imath v(z) .
$$

We want to express the system (8.5) as a nonlinear Beltrami type equation for $h$. Our computation is as follows

$$
\mathscr{F}\left(u_{x}+\imath u_{y}\right)=\mathscr{A}\left(u_{x}, u_{y}\right)+\imath \mathscr{B}\left(u_{x}, u_{y}\right)=v_{y}-\imath v_{x} .
$$

In terms of $h$ this reads as

$$
\mathscr{F}\left(\overline{h_{z}}+h_{\bar{z}}\right)=\overline{h_{z}}-h_{\bar{z}}
$$

or, equivalently

$$
\mathscr{F}\left(\overline{h_{z}}+h_{\bar{z}}\right)+\overline{h_{z}}+h_{\bar{z}}=2 \overline{h_{z}} .
$$

Next we consider the function

$$
\Psi(w) \stackrel{\text { def }}{=} \mathscr{F}(w)+w=w(2-\imath \operatorname{Arg} w)
$$


that we need to invert. First compute its complex derivatives

$$
\Psi_{w}=1+\mathscr{F}_{w}=\frac{3}{2}-\imath \operatorname{Arg} w
$$

and

$$
\Psi_{\bar{w}}=\mathscr{F}_{\bar{w}}=\frac{1}{2} \frac{w}{\bar{w}} .
$$

Hence the Jacobian determinant of $\Psi$ is positive

$$
\left|\Psi_{w}\right|^{2}-\left|\Psi_{\bar{w}}\right|^{2}=\frac{9}{4}+(\operatorname{Arg} w)^{2}-\frac{1}{4}=2+(\operatorname{Arg} w)^{2} \geqslant 2 .
$$

Therefore, the function $\Psi$ can be locally inverted. We proceed as follows

$$
\overline{h_{z}}+h_{\bar{z}}=\Psi^{-1}\left(2 \overline{h_{z}}\right)
$$

or, equivalently

$$
h_{\bar{z}}=\Psi^{-1}\left(2 \overline{h_{z}}\right)-\overline{h_{z}} .
$$

It takes a form of a nonlinear Beltrami equation

$$
h_{\bar{z}}=\mathscr{H}\left(h_{z}\right) \text {. }
$$

\section{Analysis of $\mathscr{W}^{1,2}$-solutions}

We consider here $\infty$-harmonic functions in the Sobolev class $\mathscr{W}_{\text {loc }}^{1,2}(\Omega)$. To make use of the integrating field $\mathscr{F}(w)=w(1-\imath \operatorname{Arg} w)$ we must specify a branch of the argument of $w=u_{x}+\imath u_{y}$. There are many ways to choose a measurable branch of $\operatorname{Arg} w \stackrel{\text { def }}{=} \operatorname{Arg} \nabla u$. The divergence equation at (8.1) has a meaning in the distributional sense only if both $\mathscr{A}\left(u_{x}, u_{y}\right)$ and $\mathscr{B}\left(u_{x}, u_{y}\right)$ are locally integrable. This will be easily assured by assuming that the branch of Arg $\nabla u$ lies in $\mathscr{L}_{\text {loc }}^{2}(\Omega)$.

Definition 9.1. A function $u \in \mathscr{W}_{\text {loc }}^{1,2}(\Omega)$ for which we can choose an $\mathscr{L}^{2}$-branch of $\operatorname{Arg} \nabla u$, is called a weak solution to the $\infty$-Laplace equation if

$$
\int_{\Omega}\left[\eta_{x} \mathscr{A}\left(u_{x}, u_{y}\right)+\eta_{y} \mathscr{B}\left(u_{x}, u_{y}\right)\right] \mathrm{d} x \mathrm{~d} y=0
$$

for every $\eta \in \mathscr{C}_{0}^{\infty}(\Omega)$.

Since $\infty$-harmonic functions have continuous derivatives by Savin's theorem $[\mathbf{S}]$, every $\infty$-harmonic function is a weak solution in the sense of Definition 9.1 in a neighborhood of points where the gradient does not vanish. 
From now on we assume that $\Omega$ is a simply connected domain in $\mathbb{C}$ and that $u$ is a weak solution to the $\infty$-Laplace equation. Thus the system (8.4) admits a unique (up to a constant) conjugate function $v \in$ $\mathscr{W}_{\mathrm{loc}}^{1,1}(\Omega)$.

Theorem 9.2. The mapping $h(z)=u+\imath v \in \mathscr{W}_{\mathrm{loc}}^{1,1}(\Omega)$ solves the elliptic Beltrami type equation

$$
h_{\bar{z}}=\mu(z) \overline{h_{z}}, \quad \mu(z)=\frac{\imath \theta(z)}{2-\imath \theta(z)}
$$

where $\theta(z)=\operatorname{Arg} \nabla u$. Moreover, the distortion function of $h$ is locally integrable

$$
K(z)=\frac{1+|\mu(z)|}{1-|\mu(z)|}=\frac{1}{4}\left(|\theta|+\sqrt{4+\theta^{2}}\right)^{2} \leqslant(1+|\theta|)^{2} \in \mathscr{L}_{\mathrm{loc}}^{1}(\Omega) .
$$

The Jacobian determinant of $h$ actually does not depend on the choice of $\operatorname{Arg} \nabla u$. Indeed, the first order system takes the form

or equivalently

$$
\left\{\begin{array}{l}
u_{x}+\theta u_{y}=v_{y} \\
u_{y}-\theta u_{x}=-v_{x}
\end{array}\right.
$$

$$
\operatorname{div}\left[\begin{array}{cc}
1 & \operatorname{Arg} \nabla u \\
-\operatorname{Arg} \nabla u & 1
\end{array}\right] \nabla u=0
$$

Hence

$$
J(z, h)=u_{x} v_{y}-u_{y} v_{x}=u_{x}^{2}+\theta u_{x} u_{y}+u_{y}^{2}-\theta u_{x} u_{y}=|\nabla u|^{2} \in \mathscr{L}_{\mathrm{loc}}^{1}(\Omega) .
$$

Next, let us assume that $\operatorname{Arg} \nabla u \in \mathscr{L}^{\infty}(\Omega)$, say $|\theta|<M$. For example, this is the case if $u_{y} \geqslant 0$ a.e. in $\Omega$. In this case the distortion function is bounded and $h \in \mathscr{W}_{\mathrm{loc}}^{1,2}(\Omega)$.

Corollary 9.3. If $\operatorname{Arg} \nabla u \in \mathscr{L}^{\infty}(\Omega)$ a.e. in $\Omega$ then $h$ is a $K$-quasiregular mapping, with $K=\left(1+\|\operatorname{Arg} \nabla u\|_{\infty}\right)^{2}$. In particular, $\nabla u$ may vanish only on a set of measure zero.

Proof: In fact, by Astala's area distortion theorem [As] we see that $h \in \mathscr{W}_{\text {loc }}^{1, p}(\Omega)$ with every $p<\frac{2 K}{K-1}$. Also, $h$ is Hölder continuous of exponent $\alpha=\frac{1}{K}$. Its Jacobian is positive a.e. and hence $\nabla u$ may vanish only on a set of zero measure.

Whether $\nabla u$ may vanish is not clear. For example, Aronsson $[\mathbf{A r}]$ proved that non-constant $\infty$-harmonic functions of class $\mathscr{C}^{2}(\Omega)$ have nonvanishing gradient. Based on the example (5.10), we believe that $\nabla u \neq 0$ if $u \in \mathscr{C}^{1, \alpha}(\Omega)$, with $\alpha>1 / 3$. 
Corollary 9.4. Suppose that $\theta \in \mathscr{W}_{\mathrm{loc}}^{1,2}(\Omega)$, then $u$ has locally integrable second derivatives; that is $u \in \mathscr{W}_{\mathrm{loc}}^{2,1}(\Omega)$.

Proof: It suffices to observe that the Laplacian of $u$ lies in the Hardy space $\mathscr{H}_{\mathrm{loc}}^{1}(\Omega)$. Indeed,

$$
u_{x x}+u_{y y}=u_{x} \theta_{y}-u_{y} \theta_{x}=\operatorname{det}\left[\begin{array}{cc}
u_{x} & u_{y} \\
\theta_{x} & \theta_{y}
\end{array}\right] \in \mathscr{H}_{\mathrm{loc}}^{1}(\Omega) .
$$

The Laplace equation with the Jacobian determinant in the right hand side has been investigated by Wente in 1969 [W]. His work originated intensive study of the Jacobian determinants in Hardy spaces [CLMS], [IV].

Finally we note that if $\theta \in \mathscr{W}_{\mathrm{loc}}^{1,2}(\Omega)$ a theorem of Hempel, Morris and Trudinger $[\mathbf{H M T}]$ implies that there exists $\lambda>0$ so that $\int_{\Omega} \exp \lambda \theta^{2}<\infty$. Then $h=u+\imath v$ becomes a mapping of exponentially integrable distortion

$$
|\mathrm{D} h(z)|^{2} \leqslant K(z) J(z, h), \quad K \in \operatorname{Exp}(\Omega),
$$

see $[\mathbf{I M}],[\mathbf{M M}],[\mathbf{I K M S}],[\mathbf{I K O}]$ for properties of such mappings.

Acknowledgements. Research of L. D'Onofrio, F. Giannetti, T. Iwaniec and T. Radice was supported by NSF grants DMS-0301582 and DMS-0244297 and was done while F. Giannetti and T. Radice were visiting Syracuse University.

Part of this research was done while L. D'Onofrio was a visiting researcher at SU and during this period he visited the University of Pittsburgh. They thanks SU and UP for the support and hospitality.

F. Giannetti was supported by Università degli Studi di Napoli "Federico II" in the framework of the "Programma di scambi internazionali per la mobilità di breve durata di docenti, studiosi e ricercatori".

J. Manfredi was supported by NSF grant DMS-0500983.

\section{References}

[A] L. V. Ahlfors, Conditions for quasiconformal deformations in several variables, in: "Contributions to analysis (a collection of papers dedicated to Lipman Bers)", Academic Press, New York, 1974, pp. 19-25.

[A1] L. V. Ahlfors, Quasiconformal deformations and mappings in $\mathbb{R}^{n}$, J. Analyse Math. 30 (1976), 74-97.

[Ar] G. Aronsson, On the partial differential equation $u_{x}{ }^{2} u_{x x}+$ $2 u_{x} u_{y} u_{x y}+u_{y}{ }^{2} u_{y y}=0$, Ark. Mat. 7 (1968), 395-425.

[As] K. Astala, Area distortion of quasiconformal mappings, Acta Math. 173(1) (1994), 37-60. 
[BDM $]$ T. Bhattacharya, E. DiBenedetto and J. Manfredi, Limits as $p \rightarrow \infty$ of $\Delta_{p} u_{p}=f$ and related extremal problems. Some topics in nonlinear PDEs (Turin, 1989), Rend. Sem. Mat. Univ. Politec. Torino 1989, Special Issue (1991), 15-68.

[BI] B. BOJARSKI AND T. IwANIEC, $p$-harmonic equation and quasiregular mappings, in: "Partial differential equations" (Warsaw, 1984), Banach Center Publ. 19, PWN, Warsaw, 1987, pp. 25-38.

[ClmS] R. Coifman, P.-L. Lions, Y. Meyer and S. Semmes, Compensated compactness and Hardy spaces, J. Math. Pures Appl. (9) 72(3) (1993), 247-286.

[HMT] J. A. Hempel, G. R. Morris and N. S. Trudinger, On the sharpness of a limiting case of the Sobolev imbedding theorem, Bull. Austral. Math. Soc. 3 (1970), 369-373.

[I] T. IwANIEC, $p$-harmonic tensors and quasiregular mappings, Ann. of Math. (2) 136(3) (1992), 589-624.

[I1] T. IwANIEC, On the concept of the weak Jacobian and Hessian, in: "Papers on analysis", Rep. Univ. Jyväskylä Dep. Math. Stat. 83, Univ. Jyväskylä, Jyväskylä, 2001, pp. 181-205.

[IKMS] T. Iwaniec, P. Koskela, G. Martin and C. Sbordone, Mappings of finite distortion: $L^{n} \log ^{\chi} L$-integrability, J. London Math. Soc. (2) 67(1) (2003), 123-136.

[IKO] T. Iwaniec, P. Koskela And J. Onninen, Mappings of finite distortion: monotonicity and continuity, Invent. Math. 144(3) (2001), 507-531.

[IMa] T. IwANIEC AND J. MANFREDI, Regularity of $p$-harmonic functions on the plane, Rev. Mat. Iberoamericana 5(1-2) (1989), 1-19.

[IM] T. IwANIEC AND G. MARTIN, "Geometric function theory and non-linear analysis", Oxford Mathematical Monographs, The Clarendon Press, Oxford University Press, New York, 2001.

[IS1] T. IwANIEC AND C. Sbordone, Quasiharmonic fields, Ann. Inst. H. Poincaré Anal. Non Linéaire 18(5) (2001), 519-572.

[IV] T. IwANiEc And A. Verde, A study of Jacobians in HardyOrlicz spaces, Proc. Roy. Soc. Edinburgh Sect. A 129(3) (1999), 539-570.

[JLM] P. Juutinen, P. Lindqvist and J. Manfredi, The $\infty$-eigenvalue problem, Arch. Ration. Mech. Anal. 148(2) (1999), 89-105.

[JlM1] P. Juutinen, P. Lindqvist and J. Manfredi, The infinity Laplacian: examples and observations, in: "Papers on 
analysis", Rep. Univ. Jyväskylä Dep. Math. Stat. 83, Univ. Jyväskylä, Jyväskylä, 2001, pp. 207-217.

[JLM2] P. JuUtinen, P. Lindovist and J. Manfredi, On the equivalence of viscosity solutions and weak solutions for a quasi-linear equation, SIAM J. Math. Anal. 33(3) (2001), 699-717 (electronic).

[LM] P. Lindqvist and J. Manfredi, The Harnack inequality for $\infty$-harmonic functions, Electron. J. Differential Equations 4 (1995), approx. 5 pp. (electronic).

[LM1] P. LindQVist and J. Manfredi, Note on $\infty$-superharmonic functions, Rev. Mat. Univ. Complut. Madrid 10(2) (1997), 471-480.

[MM] L. Migliaccio and G. Moscariello, Higher integrability of div-curl products, Ricerche Mat. 49(1) (2000), 151-161.

[Sa] S. Salsa, "Equazioni a derivate parziali. Metodi, modelli e applicazioni", Springer Verlag, Italia, 2004.

[S] O. Savin, $C^{1}$ regularity for infinity harmonic functions in two dimensions, Arch. Ration. Mech. Anal. 176(3) (2005), 351-361.

[W] H. C. Wente, An existence theorem for surfaces of constant mean curvature, J. Math. Anal. Appl. 26 (1969), 318-344.

Luigi D'Onofrio:

Dipartimento di Statistica e Matematica per la Ricerca Economica

Università di Napoli Parthenope

via Medina 40

80100 Napoli

Italy

E-mail address: luigi.donofrio@uniparthenope.it

Flavia Giannetti:

Dipartimento di Matematica ed Applicazioni "R. Caccioppoli"

Università di Napoli Federico II

Complesso Universitario Monte S. Angelo

via Cintia

80126 Napoli

Italy

E-mail address: giannett@unina.it

Tadeusz Iwaniec:

Department of Mathematics

Syracuse University

Syracuse, NY, 13244

USA

E-mail address: tiwaniec@mailbox.syr.edu 
Juan Manfredi:

Department of Mathematics

University of Pittsburgh

Pittsburgh PA, 15260

USA

E-mail address: manfredi@pitt.edu

Teresa Radice:

Dipartimento di Matematica ed Applicazioni "R. Caccioppoli"

Università di Napoli Federico II

Complesso Universitario Monte S. Angelo

via Cintia

80126 Napoli

Italy

E-mail address: teresa.radice@dma.unina.it

Primera versió rebuda el 31 de maig de 2005, darrera versió rebuda el 10 de gener de 2006. 